Supplementary Materials

\title{
Bio-Inspired Elastic Liquid-Infused Material for On-Demand Underwater Manipulation of Air Bubbles
}

\author{
Jianqiang Zhang, Peng Liu, Bo Yi, Zhaoyue Wang, Xin Huang, Lei Jiang, Xi Yao* \\ *Corresponding author. Email: xi.yao@cityu.edu.hk
}

The PDF file includes:

Figure S1. Fabrication and morphology characterization of eLIM.

Figure S2. Wetting and mechanical characterization of porous silicone.

Figure S3. Analysis of total interfacial energy in eLIM system.

Figure S4. Characterization of eLIM with different perfluoro-lubricant mass.

Figure S5. Schematic illustration of measuring method for the void ratio.

Figure S6. Durability characterization of eLIM underwater.

Figure S7. The changes of bubble volume in dynamic process.

Figure S8. Snapshots of releasing excessive bubbles and calculation of the critical pressure in eLIM-based pressure-limiting valve.

Other Supplementary Material for this manuscript includes the following:

Movie 1. The connectivity changes of eLIM channels under different strains.

Movie 2. Reversible tunability on bubble contact angles.

Movie 3. On-demand transportation, merging and splitting of air bubbles. 
Supplementary Movies

Movie 1 shows the bubble transportation in eLIM resulting from stretch-induced liquid redistribution and the corresponding pore connectivity. At relaxing state, two air bubbles with different curvature can keep stationary, indicating the disconnectivity of pores and channels in eLIM. Then the eLIM was taken out of water to remove bubble and stretched to $40 \%$. At stretched state, two air bubbles with different curvature can merge spontaneously, indicating the connectivity of pores and channels in eLIM.

Movie 2 shows continuous and reversible tunability on the configuration and the contact angles of depositing air bubbles.

Movie 3 shows operating multi-bubble behaviors including transportation, merging and splitting. There are four parts corresponding to figure 3 . The first part shows the transportation and merge of three bubbles on eLIM can be controlled in the way of the strain applications. The second part shows the bubble can be precisely transported from any site to the patterned site on eLIM. The third part shows two bubbles can be merged at the patterned sites on eLIM. The fourth part shows one bubble can be splited via eLIM. 


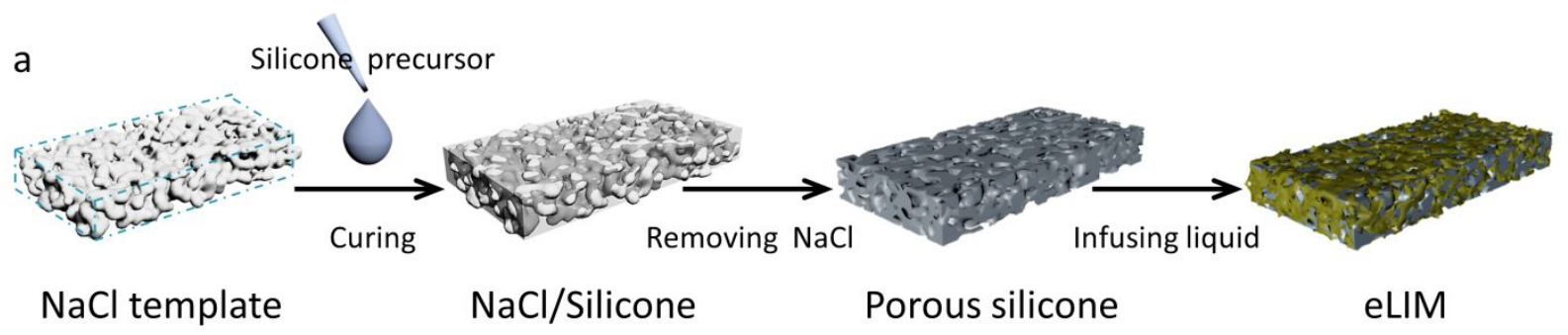

Nate

NaCl/Silicone

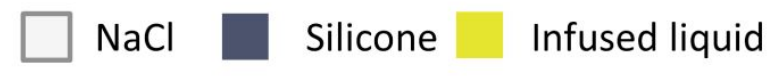

b
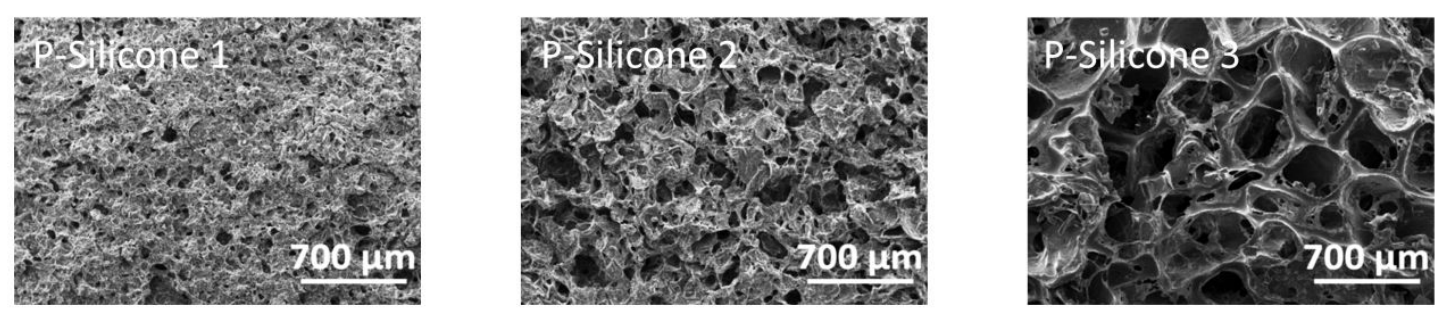

Figure S1. Fabrication and morphology characterization of eLIM. (a) Scheme showing the fabrication procedures. (b) SEM images of the porous silicone (P-Silicone) with different pore sizes. 
a

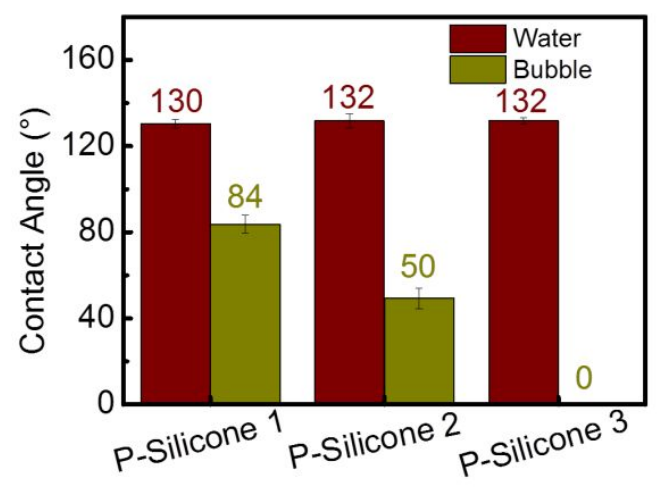

C

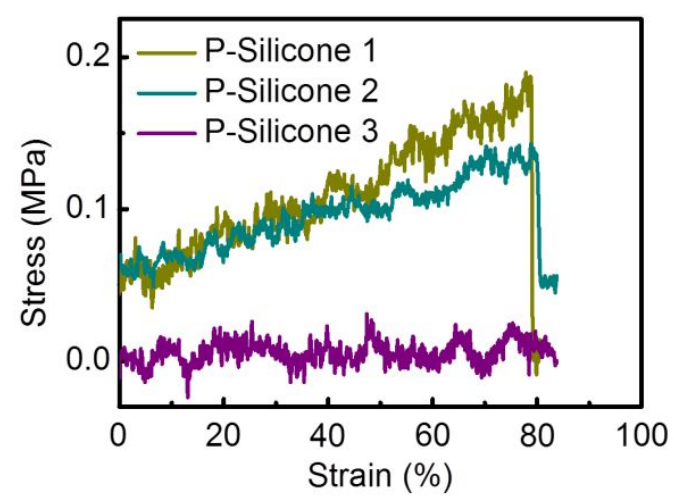

b

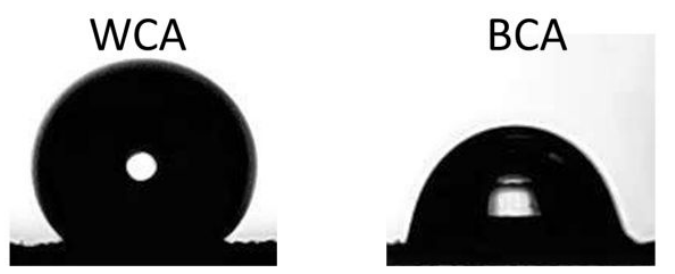

P-Silicone 1

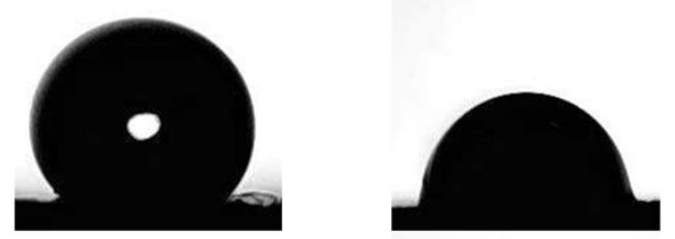

P-Silicone 2

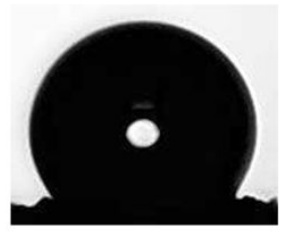

P-Silicone 3

Figure S2. Wetting and mechanical characterization of porous silicone. (a) Water contact angle (WCA) and bubble contact angle (BCA) on porous silicone and (b) corresponding optical images. (c) Strain-stress behaviors of porous silicone. 
Analysis of the total interface energy in eLIM. To keep a stable lubricant film without replacing by water in eLIM, it is necessary to select a lubricant with matchable surface tension. According to J. Aizenberg's research (ref. 16), the total interface energy in eLIM can be calculated to analyze whether infused lubricant is stable when eLIM is submerged in water. The total interfacial energy of porous silicone infused with water $\left(E_{\mathrm{w}}\right)$ or perfluoro-lubricant $\left(E_{\mathrm{o}}\right)$, is calculated in air. Moreover, underwater total interfacial energies of eLIM infused with perfluorolubricant $\left(E_{\mathrm{ow}}\right)$ also is calculated. If the eLIM system is stable underwater, the total interfacial energy must satisfy $\Delta E_{\mathrm{o}}=E_{\mathrm{w}}-E_{\mathrm{o}}>0$ and $\Delta E_{\mathrm{ow}}=E_{\mathrm{w}}-E_{\mathrm{ow}}>0$. And according to the Young's equation, the equations can be expressed as $\Delta E_{\mathrm{o}}=R\left(\gamma_{\mathrm{o}} \operatorname{con} \theta_{\mathrm{o}}-\gamma_{\mathrm{w}} \operatorname{con} \theta_{\mathrm{w}}\right)-\gamma_{\mathrm{ow}}$ and $\Delta E_{\mathrm{ow}}=$ $R\left(\gamma_{\mathrm{o}} \operatorname{con} \theta_{\mathrm{o}}-\gamma_{\mathrm{w}} \operatorname{con} \theta_{\mathrm{w}}\right)+\gamma_{\mathrm{w}}-\gamma_{\mathrm{o}}$, where $\gamma_{\mathrm{o}}, \gamma_{\mathrm{w}}, \gamma_{\mathrm{ow}}$ is the surface tension of lubricant, water and lubricant/water interface and $\theta_{\mathrm{o}}, \theta_{\mathrm{w}}, R$ is the apparent contact angles of lubricant, water, and roughness of porous silicone, respectively. As a result, we can obtain $\Delta E_{\mathrm{o}}>0$ and $\Delta E_{\mathrm{ow}}>0$, which ensure that our eLIM system is stable underwater (Figure S3).

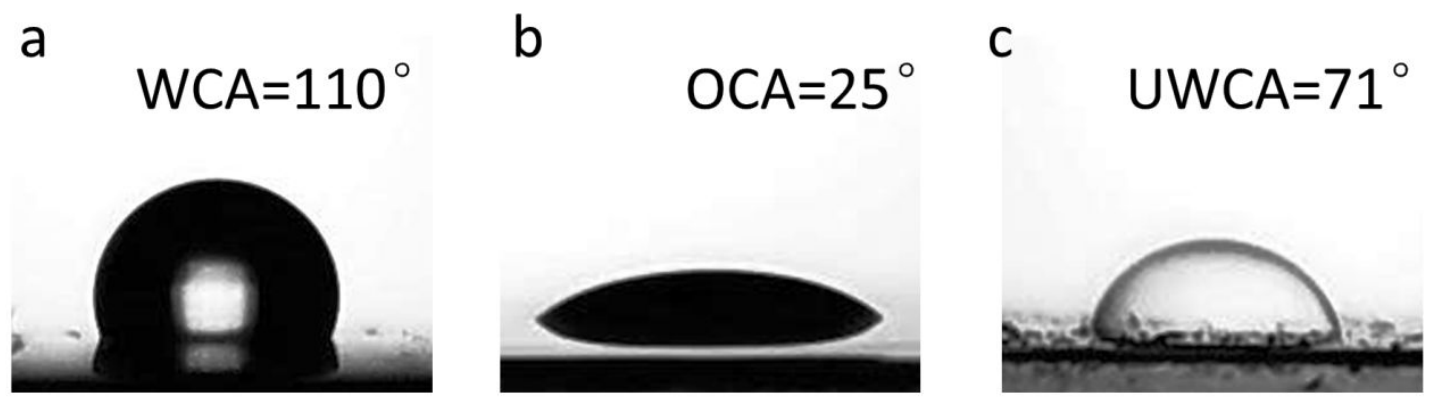

Figure S3. Analysis of total interfacial energy in eLIM system. (a) Water contact angle (WCA) of the plane silicone. (b)(c) Perfluoro-lubricant contact angle of plane silicone in air and under water, respectively. 
a

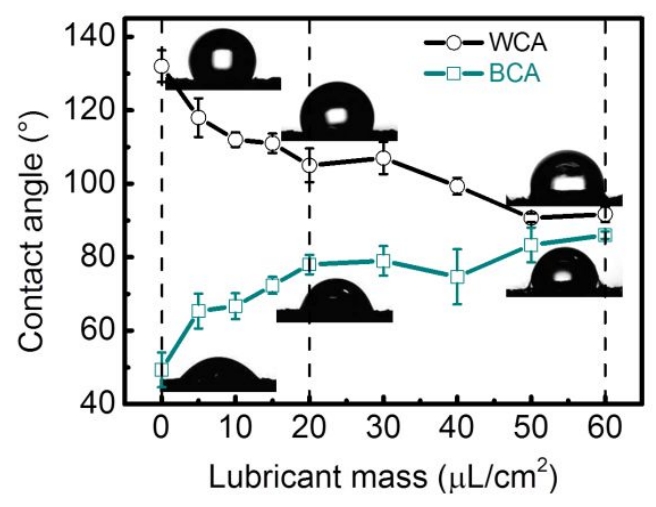

b

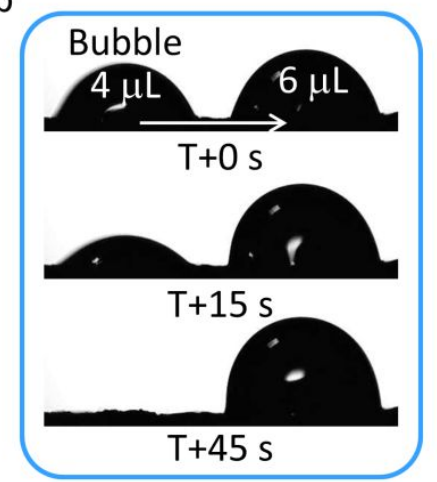

$15 \mu \mathrm{L} / \mathrm{cm}^{2}$

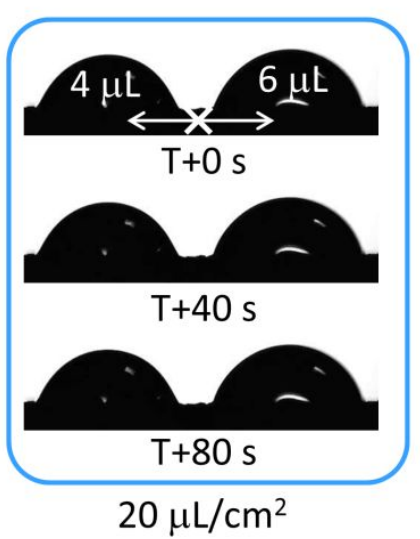

Figure S4. Characterization of eLIM with different perfluoro-lubricant mass. (a) Tuning the WCA and BCA as a function of infusing perfluoro-lubricant, respectively. In eLIM membrane with a typical thickness of $1 \mathrm{~mm}$, the infused lubricant is calculated based on the working area of the sample. (b) The lubricant-induced connectivity changes of eLIM. With increasing perfluorolubricant mass of eLIM from $15 \mu \mathrm{L} / \mathrm{cm}^{2}$ to $20 \mu \mathrm{L} / \mathrm{cm}^{2}$, the interconnected pores and channels of eLIM turn to be completely blocked by infused lubricant. So the eLIM with $20 \mu \mathrm{L} / \mathrm{cm}^{2}$ lubricant is selected to manipulate bubbles in this paper. 
Measurements of void ratio. As shown in Figure S5b, firstly, eLIM $\left(20 \mu \mathrm{L} / \mathrm{cm}^{2}\right)$ was precisely cut and then calculated the matrix volume $\left(V_{\mathrm{m}}\right)$. Secondly, this eLIM under strain was full of perfluoro-lubricant until to cover its' microstructure following to measure the total mass of eLIM. And the whole volume of perfluoro-lubricant could be calculated, which equaled to the pore volume of eLIM $\left(V_{\mathrm{p}}\right)$. The void ratio of eLIM $\left(20 \mu \mathrm{L} / \mathrm{cm}^{2}\right)$ is calculated as $V_{\mathrm{p}} / V_{\mathrm{m}}$.

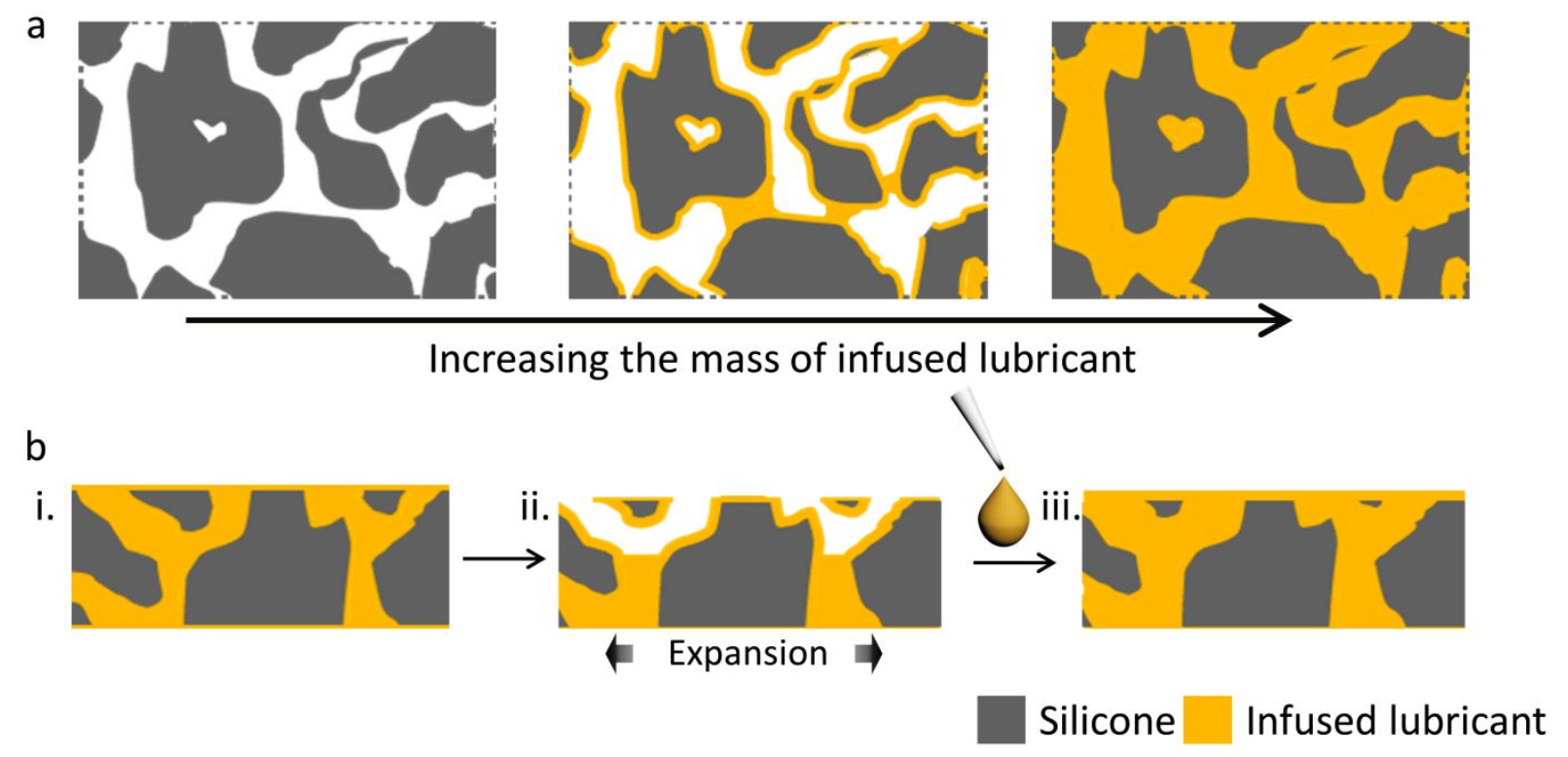

Figure S5. Schematic illustration of measuring method for the void ratio. (a) Scheme showing the infiltration progress of lubricant. (b) The method to measure the void ratio $\left(V_{\mathrm{p}} / V_{\mathrm{m}}\right)$ of eLIM with $20 \mu \mathrm{L} / \mathrm{cm}^{2}$ lubricant. 
a

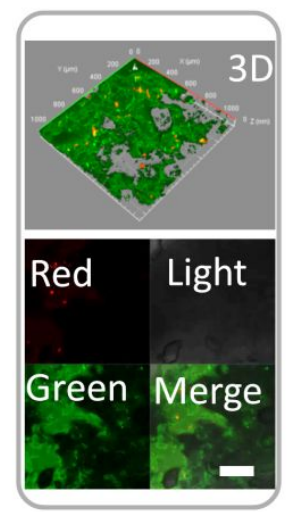

c i. Initial state

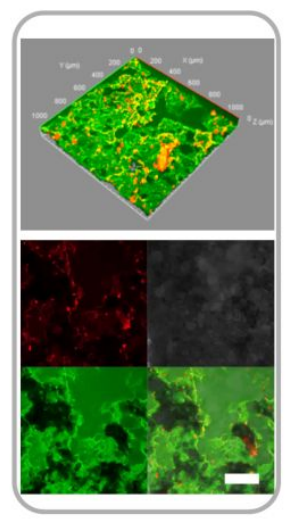

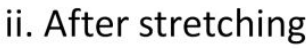
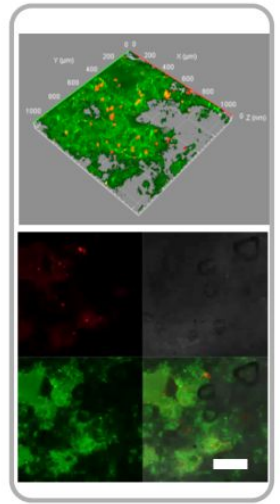

ii. After stretching

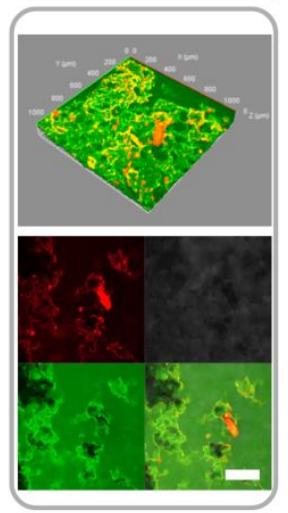

$\mathrm{b}$
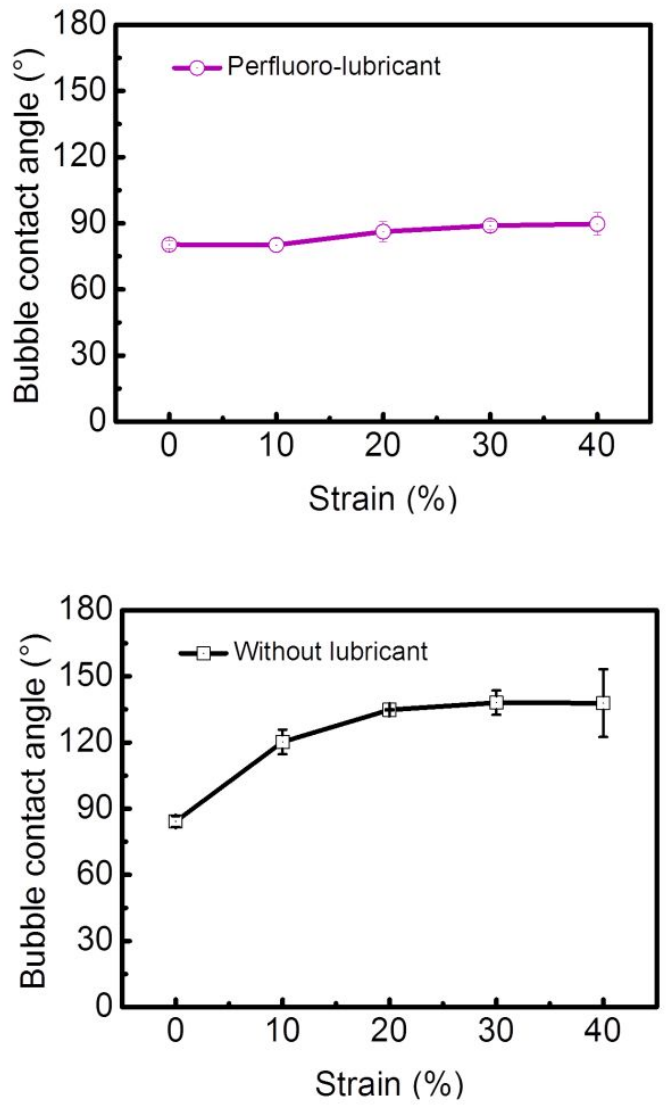

Figure S6. Durability characterization of eLIM underwater. (a) The confocal images of eLIM/water interface after repeating cycles. The eLIM (with FITC) is relaxed under water (with Rhodamine B) (i) and then is treated with stretching/relaxing operation (ii). The maximum strain is $40 \%$. The scale bar is $400 \mu \mathrm{m}$. (b) Strain-dependent BCA of eLIM after three stretching/relaxing cycles. BCA was not altered by the strain, which is consistent with the results of confocal images. (c) The confocal images of porous silicone/water interface after repeating cycles. The area of porous silicone/water interface increases after repeating cycles, which is measured by the method described above. (d) Strain-dependent BCA of porous silicone after three stretching/relaxing cycles. BCA of the porous silicone increases with increasing strain, which indicates the loss of gas affinity resulting from the impregnated water in porous silicone surface. 

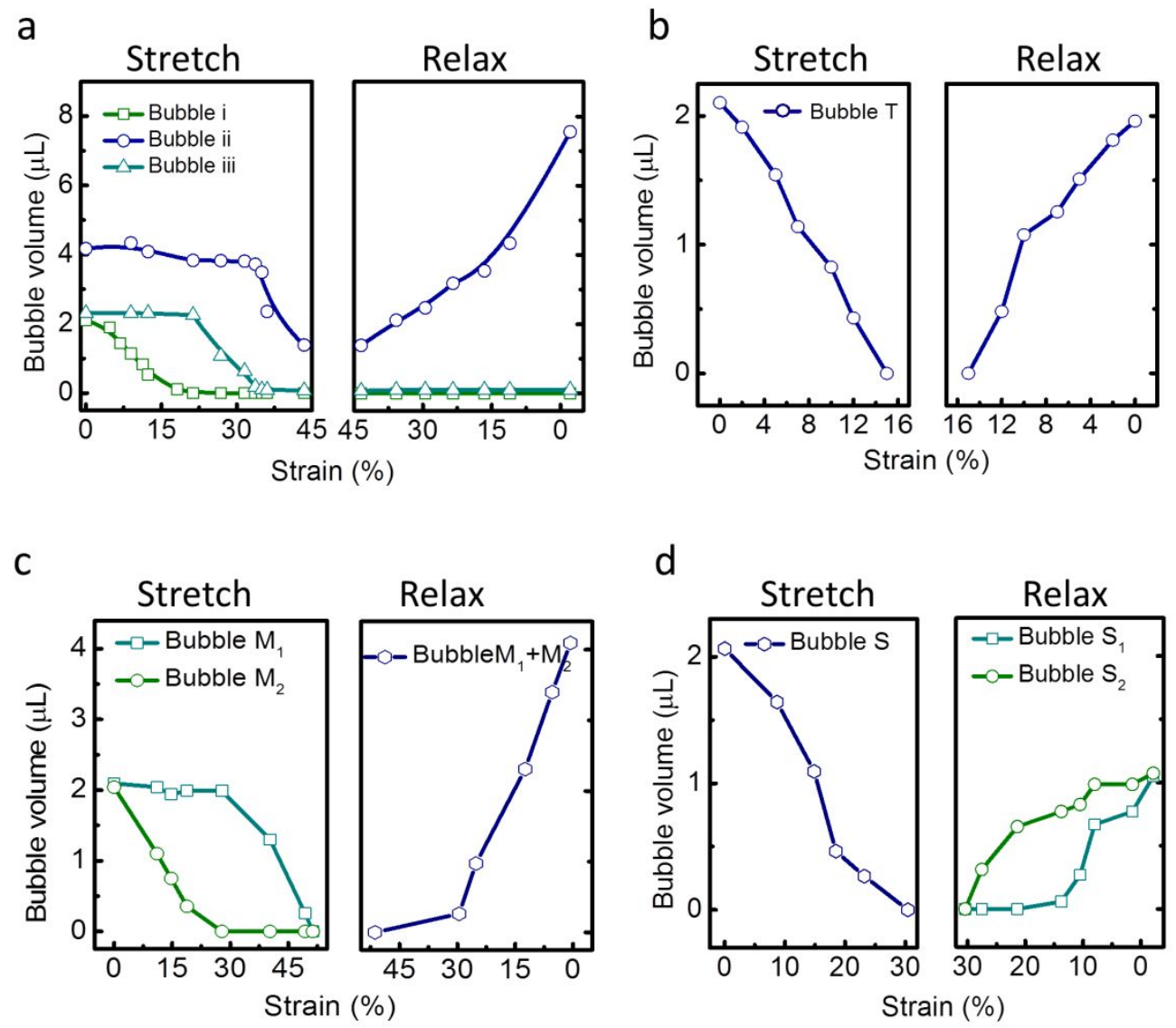

Figure S7. The changes of bubble volume in dynamic process. (a) Strain-dependent volume change of three bubbles during bubble transportation process (Figure 3a). (b) Strain-dependent volume change of bubble during bubble transportation process (Figure 3b). (c) Strain-dependent volume change of two bubbles during bubble merging process (Figure 3c). (d) Strain-dependent volume change of bubble during splitting process (Figure 3d). 
For eLIM-based pressure-limiting valve, its' critical pressure $\left(\Delta P_{\mathrm{C}}\right)$ is subject to Laplace pressure $\left(\Delta P_{\mathrm{L}}\right)$ and pressure $\left(P_{\mathrm{E}}\right)$ deriving from eLIM stretching. As shown in Supplementary Fig. 8 , the eLIM is stretched under constant flow ventilation and this deformed eLIM is simplified to a curved surface with uniform curvature. So an elastic force $F$ on the clamped edges can be expressed as $F \sim 2 \pi R h \sigma$, where $\sigma, h$, and $R$ is the stress and thickness of eLIM, the radius of the eLIM membrane mounted chamber. So $P_{\mathrm{E}}$ can be expressed as $P_{\mathrm{E}} \sim F \sin \theta /\left(\pi R^{2}\right) \sim 2 h \sigma \sin \theta / R$, where $\theta$ is the bended angle. According to the Young's modulus equation, the stress can be expressed as $\sigma=E \varepsilon \sim E(\theta / \sin \theta-1)$, where $E$ is elastic modulus and $\varepsilon$ is strain. Furthermore, Laplace pressure $\left(\Delta P_{\mathrm{L}}\right)$ is $2 \gamma_{1} / r_{\mathrm{p}}$, where $\gamma_{1}$ is the interfacial tension of infused liquid/gas and $r_{\mathrm{p}}$ is the pore radius of the eLIM membrane. Therefore, this critical pressure can be described as follow, $\Delta P_{\mathrm{C}}=\Delta P_{\mathrm{L}}+P_{\mathrm{E}} \sim 2 \gamma_{1} / r_{\mathrm{p}}+2 E h(\theta-\sin \theta) / R$.

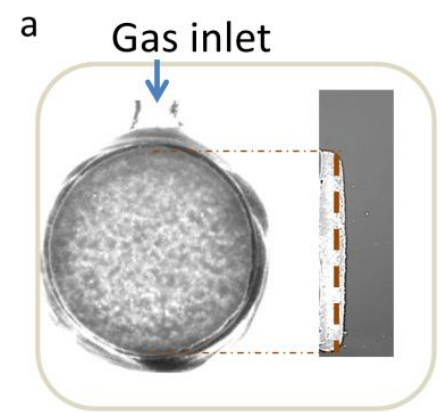

Front view Side view

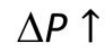

$\Delta P \downarrow$

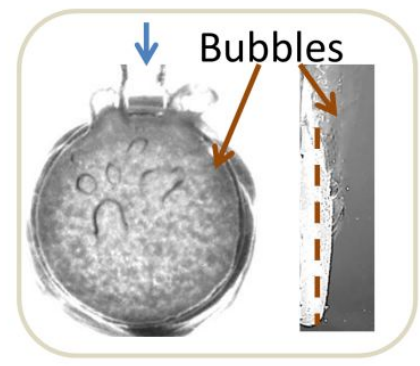

b
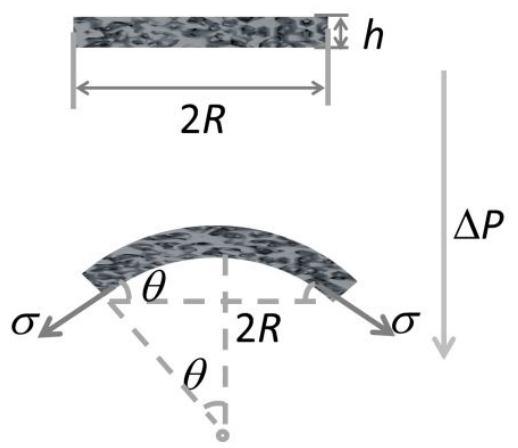

Figure S8. Snapshots of releasing excessive bubbles and calculation of the critical pressure in eLIM-based pressure-limiting valve. (a) The stretching of eLIM membrane and the release of excessive bubbles under constant flow ventilation. In order to observe the release progress of excessive bubbles, eLIM-based pressure-limiting valve was submerged into water. (b) The scheme of deformed eLIM under pressure. 